\title{
Deep-Learning Premature Contraction Localization in 12-lead ECG From Whole Signal Annotations
}

\author{
Petra Novotna $^{1}$, Tomas Vicar ${ }^{1}$, Marina Ronzhina ${ }^{1}$, Jakub Hejc $^{1,2}$, Jana Kolarova $^{1}$ \\ ${ }^{1}$ Department of Biomedical Engineering, Faculty of Electrical Engineering and Communications, \\ Brno University of Technology, Brno, Czech Republic \\ ${ }^{2}$ Department of Pediatric, Children's Hospital, University Hospital Brno, Brno, Czech Republic
}

\begin{abstract}
Since common electrocardiography (ECG) diagnostics approaches are time-consuming and arrhythmia-type sensitive, deep-learning methods are state-of-the-art for their detection accuracy. However, premature ventricular contractions' (PVC) localization via common deep-learning approaches requires large training set, therefore Multiple Instance Learning (MIL) framework was applied, where model is trained from whole-signal annotations. Proposed MIL framework is based on 1D Convolutional Neural Network (CNN), with global max-pooling in the last layer. The detection of PVCs' positions was done by the peak detector with specified parameters - threshold, minimal distance and peak prominence. Our method was tested on database containing 1590 ECGs, including 672 signals with PVCs. Dice coefficient reaches 0.947 . This simple deep-learning method for the localization of PVC achieves a promising performance while being trainable from the whole-signal annotations instead of positional labels.
\end{abstract}

\section{Introduction}

Premature ventricular contraction (PVC) is one of the commonly diagnosed arrhythmias. In the last decade, multiple approaches were applied on PVC detection in ECG signal. One of the most intuitive PVC detectors are those based on the decision rules, where the rhythm and morphological features are calculated from the beats and PVCs are identified by the feature assessment [1]. Another simple method is a template matching, where a set of appropriate beat's templates is required. Malek et al. in their study describe an improved template matching combined with QRS features analysis and assessment of several different correlation coefficients [2]. Gordon et al. use a sofisticated deep learning technique - convolutional autoencoder - for automatic feature extraction and simple random forest for final detection of PVC beats [3]. Contrary to aforementioned approaches, no manually defined features nor selec- tion of the most appropriate features are needed.

Since common diagnostics approaches are timeconsuming and arrhythmia-type sensitive, deep-learning methods are state-of-the-art for their detection accuracy, which can even surpass qualified medical experts. However, PVCs localization via common deep-learning approaches requires large training set including detailed PVCs annotation. Here we present model which has no such limitation. It localizes PVCs based on the whole signal annotation (i.e. ECG signal with/without PVC(s)). For this weakly-supervised training, Multiple Instance Learning (MIL) framework was applied. MIL in combination with deep learning was previously used for e.g. retinal image quality estimation in [4]. Proposed MIL framework is based on 1D Convolutional Neural Network (CNN) trained with global annotations with no PVC nor normal QRS positions required.

\section{Methods}

The presented PVC detection method consists of three main steps: 1) ECG signal classification (ECG with/without PVCs) based on global annotation, 2) MIL based feature signal calculation, 3) PVC complexes localization using peak-detection via MIL feature signal alignment.

\subsection{ECG data-set}

Publically available ECG database from China Physiological Signal Challenge 2018 (CPSC2018) [5] was used. In this database, only whole-signal annotations are available. From original database, only signals labeled as Normal or PVC were used. The data-set contains 1590 ECGs altogether, out of which 672 are labeled as signal with PVCs. To test the proposed PVC detector, we labeled position of PVCs manually. The data-set was split into training and testing subsets in 8:2 ratio. ECGs were divided by the standard deviation from the training dataset. 


\subsection{Classification network architecture}

For MIL framework, CNN architecture was applied. Proposed architecture based on a residual neural network (ResNet) [6], which has been modified form image to signal processing by replacing all $2 \mathrm{D}$ operation by its $1 \mathrm{D}$ equivalents. Network architecture is depicted on Figure 1.

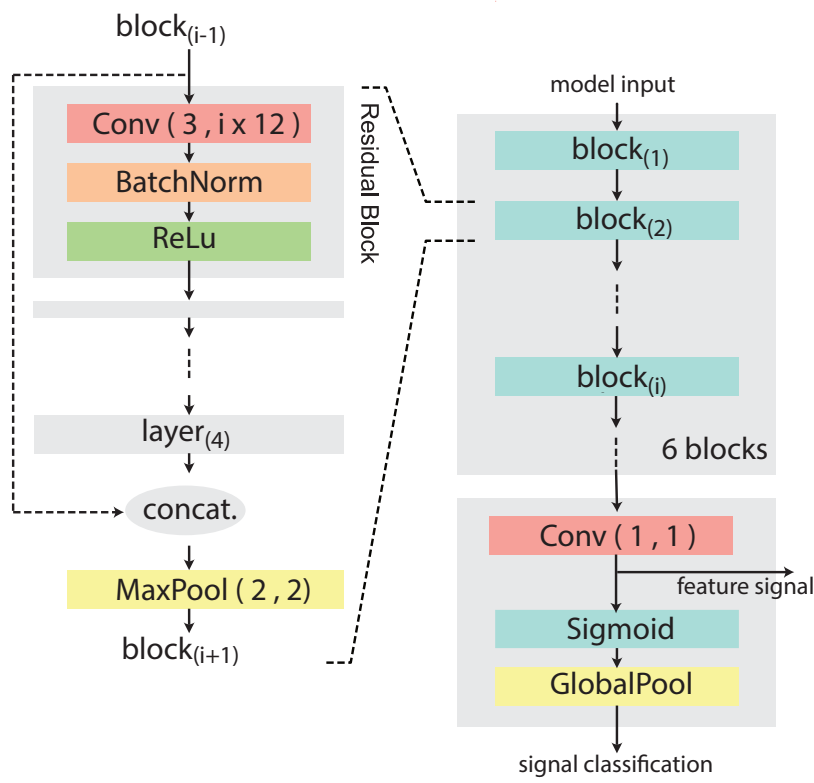

Figure 1: Architecture of proposed model. Conv $(3, i \times 12)$ - convolution layer with filter size 3 and $i \times 12$ filters; $\operatorname{Max} \operatorname{Pool}(2,2)$ - $\max$ pooling with filter size 2 and stride 2 .

\subsection{MIL framework}

By processing input ECG via several convolutional and pooling layers, subsampled MIL feature signal (position likelihood) of a variable length (bag of instances) is obtained and is projected to a single output label by global max-pooling followed by standard weighted cross-entropy loss (WCE). The MIL feature signal $p_{i}$ (output of sigmoid activation function) indicates, whether and how much this part of ECG contributed to final prediction. MIL WCE loss is:

$$
\begin{aligned}
& W C E_{M I L}=w_{\text {pos }} t \log \left(\max _{i} p_{i}\right)+ \\
& \quad+w_{n e g}(1-t) \log \left(1-\max _{i} p_{i}\right),
\end{aligned}
$$

where $t$ is a binary signal label, $w_{\text {pos }}$ and $w_{\text {net }}$ are weights for positive and negative class, respectively. More frequent class has proportionally lower weight. Thus, high feature values correspond to PVC area in ECG (see Figure 2). The maps of PVC likelihood are further processed to get PVC locations.

\subsection{PVC localization}

The detection of PVCs' positions was done by the peak detector with specified parameters - threshold, minimal distance and peak prominence [7]. Optimal parameters' settings were estimated via Python implementation [8] of Bayesian Optimization [9]. The parameters' optimisation bounds were set to: minimum - maximum of all PVC likelihood maps; 0 - min-max range for peak prominence; 0 $2 \mathrm{~s}$ for minimal peak distance.

\subsection{Training details and implementation}

The model was optimised with Adam optimizer $\left(\beta_{1}=\right.$ $\left.0.9 ; \beta_{2}=0.999\right)[10]$ with decoupled weight decay regularization $\left(\lambda=10^{-5}\right)[11]$. Initial learning rate was set to 0.001 and was multiplied by 0.1 every 50 epochs. Model was trained with weighted cross-entropy loss, where weights are inversely proportional to frequency of PVCs in the training dataset. The mini-batch size was set to 32 and weights were initialised with Xavier initialisation [12]. Data augmentation consisted of random multiplication by 0.3 of each lead, stretching the signals by 20 $\%$ and circular shifting of signal part. Zero-padding was applied for signals to fit the batch size.

Code is available at: https://github.com/ PetraNovotna/MIL_based_PVC_localization.

\section{Results}

Correctness of PVC detection depends on the performance of the classification model as well as precision of peak detection in MIL feature signal. ECG classification model reached accuracy on test dataset 0.961 and 0.967 for average pooling and max pooling, respectively. The accuracy is comparable with the results of another recent studies with CNN classification (e.g. 0.979 [13], 0.979 [14]).

An example of MIL feature signal and detected PVC locations can be seen in Figure 2. Yellow to red parts of feature signal refer to ECG areas contributing significantly to final PVC classification.

Results of PVC localization are summarized in Table 1. Both model configurations reached satisfactory results when comparing with another reports regarding recall, precision and Dice coefficient. It should be noticed, that QRS positions are not available for initial ECGs. In contrast to previously published studies (Table 1), the QRS positions are not required to train the presented model. Only PVC positions were manually detected for test data. Therefore, true negatives and, consequently, detection accuracy were not calculated. Straightforward comparison of the methods is not possible since different databases (in terms of size, ECG duration, acquisition setup, present pathologies) 

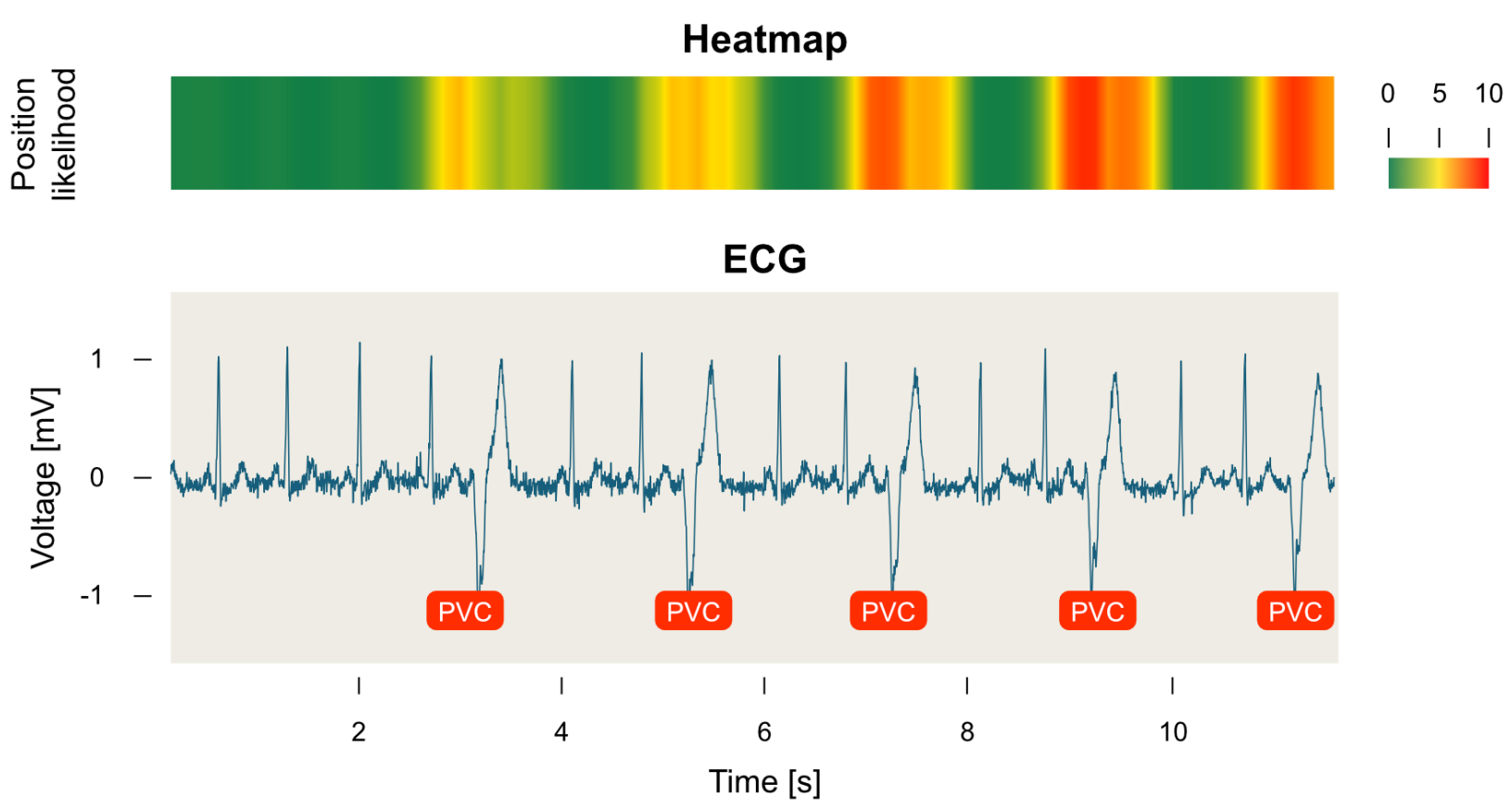

Figure 2: MIL feature signal and PVC beats detection. PVC position likelihood is represented by color scale, where green refers to uninteresting areas and yellow-to-red parts refer to ECG areas contributing significantly to final PVC classification.

Table 1: Comparison of results with previously published approaches; * regarding test database; ** calculated as ratio of all accurately detected QRS (available for classification of previously detected QRS complexes into two categories).

\begin{tabular}{|c|c|c|c|c|c|c|}
\hline Author & Data & Method & Rec & Prec & Dice & Acc** \\
\hline $\begin{array}{l}\text { Lustgarten } \\
\text { [1] }\end{array}$ & ICM, Holter (PVC,Other) & $\begin{array}{l}\text { QRS features, } \\
\text { rule-based detection }\end{array}$ & $\begin{array}{l}0.744 \text { to } \\
0.752 *\end{array}$ & $\begin{array}{l}0.688 \text { to } \\
0.759^{*}\end{array}$ & $\begin{array}{l}0.715 \text { to } \\
0.755^{*}\end{array}$ & $\begin{array}{l}0.991 \text { to } \\
0.992 *\end{array}$ \\
\hline Malek [2] & $\begin{array}{l}\text { MIT-BIH, INCART, QT, } \\
\text { Fantasia (PVC,Normal) }\end{array}$ & Template matching & $\begin{array}{l}0.809 \text { to } \\
0.939^{*}\end{array}$ & - & - & $\begin{array}{l}0.979 \text { to } \\
0.998^{*}\end{array}$ \\
\hline $\begin{array}{l}\text { Gordon } \\
{[3]}\end{array}$ & MIT-BIH (PVC,Other) & $\begin{array}{l}\text { Autoencoder, } \\
\text { Random forest }\end{array}$ & 0.865 & 0.623 & 0.724 & 0.878 \\
\hline Proposed & CPSC2018 (PVC, Normal) & MIL - avg-pool & 0.865 & 0.890 & 0.877 & - \\
\hline Proposed & CPSC2018 (PVC, Normal) & MIL - max-pool & 0.946 & 0.948 & 0.947 & - \\
\hline
\end{tabular}

were used. Model with max pooling layer outperformed that with average pooling layer by approx. $7 \%$.

\section{Discussion and Conclusions}

Our method was tested on database containing 1590 ECGs, including 672 signals with PVCs. Dice coefficient reaches 0.947 for model with max pooling layer. The main advantage of our method is in it's potential applicability on detection of various types of abnormalities without significant, arrhythmia-specific, changes in model configuration. This simple deep-learning method for the localization of PVC achieves a promising performance while be- ing trainable from the whole-signal annotations instead of positional labels. Another benefit of the method is that no previously detected QRS positions are required. From practical point of view, MIL framework useful in reducing the time needed for detailed ECG analysis and improved the diagnostic process.

\section{References}

[1] Lustgarten DL, Rajagopal G, Reiland J, Koehler J, Sarkar S. Premature ventricular contraction detection for long-term monitoring in an implantable cardiac monitor. Pacing and Clinical Electrophysiology 2020;43(5):462-470.

[2] Malek AS, Elnahrawy A, Anwar H, Naeem M. Automated 
detection of premature ventricular contraction in ECG signals using enhanced template matching algorithm. Biomedical Physics Engineering Express 2020;6(1):015024.

[3] Gordon M, Williams C. Pvc detection using a convolutional autoencoder and random forest classifier. In Pacific symposium on biocomputing. 2019; 42-53.

[4] Costa P, Campilho A, Hooi B, Smailagic A, Kitani K, Liu S, Faloutsos C, Galdran A. Eyequal: Accurate, explainable, retinal image quality assessment. In 2017 16th IEEE International Conference on Machine Learning and Applications (ICMLA). IEEE, 2017; 323-330.

[5] Liu F, Liu C, Zhao L, Zhang X, Wu X, Xu X, Liu Y, Ma C, Wei S, He Z, et al. An open access database for evaluating the algorithms of electrocardiogram rhythm and morphology abnormality detection. Journal of Medical Imaging and Health Informatics 2018;8(7):1368-1373.

[6] He K, Zhang X, Ren S, Sun J. Deep residual learning for image recognition. In Proceedings of the IEEE conference on computer vision and pattern recognition. 2016; 770-778.

[7] Ahn J, Choi MH, Kim K, Senok SS, Cho DiD, Koo Ki, Goo Y. The advantage of topographic prominence-adopted filter for the detection of short-latency spikes of retinal ganglion cells. The Korean Journal of Physiology Pharmacology 2017;21(5):555-563.

[8] Nogueira F. Bayesian Optimization: Open source constrained global optimization tool for Python, 2014-. URL https://github.com/fmfn/ Bayesianoptimization

[9] Snoek J, Larochelle H, Adams RP. Practical bayesian optimization of machine learning algorithms. In Advances in neural information processing systems. 2012; 2951-2959.

[10] Kingma DP, Ba J. Adam: A method for stochastic optimization. arXiv preprint arXiv14126980 2014;.

[11] Loshchilov I, Hutter F. Decoupled weight decay regularization. arXiv preprint arXiv171105101 2017;

[12] Glorot X, Bengio Y. Understanding the difficulty of training deep feedforward neural networks. In Proceedings of the thirteenth international conference on artificial intelligence and statistics. 2010; 249-256.

[13] Zhao Z, Wang X, Cai Z, Li J, Liu C. Pvc recognition for wearable ecgs using modified frequency slice wavelet transform and convolutional neural network. In 2019 Computing in Cardiology (CinC). 2019; 1-4.

[14] Ullah A, Anwar SM, Bilal M, Mehmood RM. Classification of arrhythmia by using deep learning with 2-d ecg spectral image representation. Remote Sensing 2020;12(10):1685.
Address for correspondence:

Petra Novotna

Department of Biomedical Engineering, Faculty of Electrical Engineering and Communication, Brno University of Technology, Technicka 12, 61600 Brno, Czech Republic. novotnap@vutbr.cz 